\title{
SUPLEMENTACIÓN CON AMINOÁCIDOS DE CADENA RAMIFICADA EN LA PROGRESIÓN DE LA HEPATITIS CRONICA
}

\section{SUPPLEMENTATION WITH BRANCHED-CHAIN AMINO ACID ON THE PROGRESSION OF CRONIC HEPATITIS}

\author{
Jorge Mario Cruz Amaya ${ }^{1}$, Melina Flórez Cuadros², William Narváez Solarte ${ }^{3}$
}

\begin{abstract}
${ }^{1}$ DMV, Ph.D., Docente. Universidad Nacional de Colombia, Sede Medellín, Facultad de Ciencias Agrarias. Calle 59 ${ }^{A}$ No. 63-40, Grupo de investigación en biodiversidad y genética molecular BIOGEM, e-mail: jmcrusa@unal.edu.co; ${ }^{2}$ MVZ, cPh.D. Universidad de Nebraska, e-mail: melinaflorezcuadros@huskers.unl.edu; ${ }^{3}$ ZOOT., Ph.D., Docente. Universidad de Caldas, Facultad de Ciencias Agropecuarias. Calle 65 No. 26-10, Manizales, Colombia, e-mail: wnarvaez@ucaldas.edu.co
\end{abstract}

Rev. U.D.C.A Act. \& Div. Cient. 19(1): 155-160, Enero-Junio, 2016

\section{RESUMEN}

La malnutrición calórico-proteica es una condición frecuente en la falla hepática. El objetivo fue evaluar el efecto de la suplementación con aminoácidos de cadena ramificada (ACR), en la dieta de ratas con hepatitis crónica. Se generó daño hepático, aplicando tetracloruro de carbono (CCL4), durante diez semanas, en todos los tratamientos, excepto en el control. Las unidades experimentales fueron asignadas aleatoriamente a seis tratamientos: valina, leucina, isoleucina, los tres ACR, CCL 4 y control, con cinco repeticiones por tratamiento y tres animales por unidad experimental. Se midió la concentración sérica de albúmina. Las lesiones tisulares histopatológicas fueron evaluadas, asignando un valor de cero a tres a variables, como infiltración celular, vacuolización y fibrosis. Se monitoreó la ganancia de peso, el consumo de alimento y los signos relacionados con daño hepático. Las muestras fueron tomadas en la semana 6, 8 y 10 del experimento. Se realizó un análisis de varianza, fijando una significancia de $\mathrm{p}<0,05$ y la prueba de comparación múltiple de Tukey, en la presencia de diferencia estadísticamente significativa. El suplemento de la dieta con ACR no alteró la progresión de la fibrogénesis; sin embargo, las ratas bajo el tratamiento valina, mostraron un peso significativamente superior. Los animales en los tratamientos leucina y valina exhibieron un consumo de alimento significativamente superiores. En ningún tratamiento se observaron signos de insuficiencia hepática. Se concluye que la suplementación con ACR valina y leucina podrían tener un efecto benéfico en el desbalance proteico, que se presenta en la enfermedad hepática crónica.

Palabras clave: Enfermedad hepática, aminoácidos de cadena ramificada, desnutrición proteica.

\section{SUMMARY}

Malnourishment is a common condition of hepatic failure. Our objective was to evaluate the effect of supplementation with branched-chain amino acid (BCAA) in the diet of rats with chronic hepatitis. Liver damage was generated using carbon tetrachloride $\left(\mathrm{CCl}_{4}\right)$ for ten weeks in all treatments except control. Experimental units were randomly assigned to six treatments: valine, leucine, isoleucine, three $\mathrm{BCAA}, \mathrm{CCl}_{4}$ and control, five replicates per treatment, and three animals per experimental unit. Serum albumin concentrations were compare. Tissue injury level was histopathologically graded from zero to three looking for disturbances such as cellular infiltration, fibrosis, and vacuolization. Also, weight gain, food intake, and liver damage signs were monitored. Samples were taken at week 6,8 , and 10 . When the effect of treatment was significant $(p<0.05)$ in the analysis of variance, multiple comparisons were adjusted with Tukey-Kramer method. The dietary supplement with BCAA does not alter the progression of fibrogenesis. However, rats under valine treatment showed significantly more weight. Animals in leucine and valine treatments exhibited significantly higher food intake. No signs of liver failure were observed in any treatment. As conclusion, valine and leucine supplementation could have a positive effect in the protein imbalance present in the chronic liver disease.

Key words: Chronic hepatitis, branched chain amino acids, protein malnutrition.

\section{INTRODUCCIÓN}

La malnutrición calórico-proteica es una condición frecuente, compleja y multifactorial en la falla hepática, que se presenta hasta en un $90 \%$ de los pacientes con cirrosis (Larsen, 
2008) y está fuertemente asociada a mayor morbilidad y mortalidad (Khanna \& Gopalan, 2007).

La disminución en el consumo de proteínas, causado por la sensación de saciedad, la alteración del sentido del gusto y la incapacidad del hígado para metabolizar glucosa, puede desarrollar un estado catabólico y caquexia (Charton, 2006). De otra parte, existe en estos pacientes una alteración en la relación de los aminoácidos de cadena ramificada (ACR) y los aminoácidos aromáticos (AAA), que se caracteriza por una disminución notable de los primeros (Larsen, 2008; Tajiri \& Shimizu, 2013), siendo este tipo de aminoácidos un sustrato importante para la detoxificación cerebral e inhibición de la proteólisis del músculo esquelético (Kondrup, 2006).

La desnutrición proteica en la cirrosis esta usualmente caracterizada por la reducción en la albúmina sérica y la disminución del volumen del músculo esquelético. Existe una correlación altamente significativa entre los niveles plasmáticos de albúmina y los ACR, donde los niveles bajos de ACR en plasma van asociados a hipoalbuminemia (Yoshida et al. 1989).

Del mismo modo, el hígado juega un papel importante en el metabolismo energético. Los pacientes con cirrosis presentan un deterioro en la acumulación del glucógeno, debido a la atrofia del órgano y, por lo tanto, desarrollan estados catabólicos severos, cuando se presenta ayuno (Nakaya et al. 2007).

Las hepatopatías crónicas, con frecuencia, son incurables, ya que los tratamientos instaurados no detienen la progresión de la enfermedad a cirrosis y la consecuente falla hepática. La terapia, se realiza dirigida al control de los síntomas que se van desarrollando y en evitar las complicaciones secundarias. Las modificaciones dietéticas, con base en el balance de la calidad y la cantidad de proteína, se mantienen, como uno de los pilares del tratamiento para la enfermedad hepática crónica (García et al. 2007; Tajiri \& Shimizu, 2013).

El trasplante hepático es la única alternativa de supervivencia a largo plazo cuando existe cirrosis, pero debido a la poca disponibilidad de órganos y los altos costos de la cirugía, no está ampliamente disponible (García et al. 2007). En la actualidad, la terapia de la falla hepática, tanto en animales como en el hombre, está muy lejos de haber alcanzado un objetivo óptimo y sigue planteando serios retos en la investigación (Muddu et al. 2007).

La terapia con ACR en pacientes con fallo hepático, se ha intentado en el pasado y, en la actualidad, existen conceptos contradictorios al respecto. Algunos autores no han encontrado efectos benéficos (Charton, 2006), mientras que otros han observado que mejoran la relación de ACR/AAA en plasma (Nakaya et al. 2002), la ganancia de peso, el aumento de la albúmina plasmática y la calidad de vida (Moriwaki et al. 2004; Chen et al. 2015) y disminuyen los signos de encefalopatía (Marchesini et al. 2005; Tajiri \& Shimizu, 2013).

El objetivo de este trabajo fue evaluar el efecto de la suplementación en la dieta con ACR, en ratas con enfermedad hepática crónica.

\section{MATERIALES Y MÉTODOS}

El trabajo de campo, se llevó acabo en el bioterio de la Universidad de Caldas, ubicado en Manizales, Colombia, a $2.150 \mathrm{msnm}$, con una temperatura promedio de $20^{\circ} \mathrm{C}$ y humedad relativa de $78 \%$. Las dietas evaluadas fueron elaboradas en la Unidad Tecnológica de Alimentos y los análisis histopatológicos y sanguíneos en los laboratorios de Patología Veterinaria y Patología Clínica de la misma institución. La presente investigación fue avalada por el comité de ética para la experimentación con animales de la Universidad de Caldas, acta número 2 de abril 28, 2009.

Se utilizaron 81 ratas (Rattus norvegicus), línea Wistar, con un peso inicial aproximado de $257 \mathrm{~g}$ y ocho meses de edad, procedentes del bioterio de la Universidad de Caldas. Se distribuyeron en 25 cajas plásticas trasparentes de $40 \mathrm{~cm}$ de largo, 30 de fondo y 50 de alto (Vanyplas ${ }^{\circledR}$ ), con $500 \mathrm{~g}$ de cascarilla de arroz, como cama, con recambio dos veces a la semana.

El alimento, se dispuso ad libitum. Hasta los siete meses de edad recibieron una dieta balanceada, que contenía $18 \%$ de proteína bruta, $4 \%$ de extracto etéreo, $5 \%$ de fibra bruta, $1 \%$ de calcio y $0,8 \%$ de fósforo. Después de este periodo, los animales tuvieron una etapa de adaptación a la dieta experimental, con un cambio gradual de ocho días, hasta recibir el $100 \%$ de la dieta experimental básica, la cual, no contenía $\mathrm{ACR}$, ni preservantes.

Los animales, se pesaron semanalmente desde el inicio del periodo experimental, en una báscula digital. De igual manera, en una ficha de seguimiento, se realizó el registro del consumo de alimento diario, la mortalidad y los signos clínicos observados en cada tratamiento.

Como dieta básica experimental, se utilizó la AIN 76, descrita por el American Institute of Nutrition para ratas de laboratorio (AIN, 1977), que contiene por cada kilogramo: $500 \mathrm{~g} /$ sacarosa, $200 \mathrm{~g} /$ caseína, $150 \mathrm{~g} /$ fécula de maíz, $50 \mathrm{~g} /$ celulosa, $50 \mathrm{~g} /$ aceite de maíz, $30 \mathrm{~g} /$ metionina, $20 \mathrm{~g} /$ cloruro de colina. A esta dieta, se adicionó leucina, isoleucina y valina, para formar los diferentes tratamientos.

Una vez formuladas las dietas experimentales, se procedió a su elaboración en una mezcladora de tipo cilíndrica, con 
50L de capacidad, adicionando todos los ingredientes y $6 \%$ de agua a $60^{\circ} \mathrm{C}$. Se mezclaron los ingredientes durante 15 min; posteriormente, la mezcla se pasó por un molino con criba (Omega $\left.{ }^{\circledR}\right)$, formando los pellets, que fueron secados en un horno, a una temperatura promedio de $55^{\circ} \mathrm{C}$, durante dos horas y, luego, almacenados en el congelador, a $-4^{\circ} \mathrm{C}$, hasta su uso.

Superado el periodo de adaptación a la dieta, se indujo el daño hepático, en todos los animales, con la aplicación subcutánea de CCL4, menos en el grupo control, según el protocolo de Proctor \& Chatamra (1984). EL CCl4, se preparó disuelto en aceite de oliva, en una proporción de 1:1. Esta mezcla, se aplicó dos veces por semana, a las dosis de $1 \mathrm{~mL} /$ $\mathrm{kg}$ de peso corporal, por vía subcutánea, durante diez semanas.

A la sexta, octava y décima semana de aplicar el CCL4, se muestreó un animal por unidad experimental, para observar los efectos de los tratamientos. Los animales fueron sacrificados con una sobre dosis de isofluorano (Isorane Lab.
Abbot $(1)$, según lo establecido por la American Veterinary Medical Association (2007). Una vez logrado el paro cardiorrespiratorio, se tomó la muestra de sangre intracardiaca sin anticoagulante y una muestra de tejido hepático, utilizando, como conservante, formol al $10 \%$. A la necropsia del animal, se registraron los hallazgos macroscópicos.

En el Laboratorio de Patología Clínica, las muestras de sangre se centrifugaron a 2000rpm, durante $15 \mathrm{~min}$, para la obtención de suero y la medición de albúmina. Las muestras de tejido hepático fueron coloreadas con hematoxilina-eosina. La lectura de las placa, se realizó teniendo en cuenta el grado de actividad inflamatoria, vacuolización y fibrosis, asignando una escala de cero, a tres a cada variable, donde cero no presenta actividad y tres presenta la actividad máxima, adaptando el sistema marcador METAVIR (Theise, 2007), especialmente diseñado, para pacientes con hepatitis.

Los animales, se distribuyeron en 6 tratamientos, en un diseño irrestrictamente al azar, formado por cinco repeticiones y tres animales por unidad experimental (Tabla 1).

Tabla 1. Tratamientos experimentales, cantidad en gramos de aminoácidos de cadena ramificada, suplementados por cada $100 \mathrm{~g}$ de dieta experimental.

\begin{tabular}{|l|l|l|l|l|}
\hline Tratamiento & $\mathrm{CCl}_{4}$ & leucina & isoleucina & valina \\
\hline T0 CCL4 & + & 1,620 & 1,000 & 1,228 \\
\hline T1 ACR & + & 2,694 & 1,522 & 1,832 \\
\hline T2 leucina & + & 2,994 & 0,922 & 1,132 \\
\hline T3 isoleucina & + & 1,494 & 1,672 & 1,132 \\
\hline T4 valina & + & 1,494 & 0,922 & 2,007 \\
\hline T5 control & - & 1,620 & 1,000 & 1,228 \\
\hline
\end{tabular}

$(+)=1 \mathrm{mLCCL}_{4}$ de peso vivo durante diez semanas, subcutáneo, durante 10 semanas; $(-)=$ no aplica.

Se realizó un análisis de varianza (ANOVA), fijando la $p<0,05$ y se utilizó la prueba de comparación múltiple de Tukey, en la presencia de diferencia estadísticamente significativa.

\section{RESULTADOS Y DISCUSIÓN}

En este trabajo, ningún tratamiento con adición de ACR alteró la progresión de la fibrogénesis ni el daño hepático; no se observaron diferencias estadísticas significativas en la evaluación histopatológica de las variables vacuolización, infiltración celular y fibrosis en los diferentes tratamientos. En general, las lesiones histopatológicas más notorias observadas fueron vacuolización acompañada de degeneración grasa y fibrosis centrolobulillar o puentes fibrocitos de comunicación porto-central, que se manifestaron de manera progresiva y con mayor intensidad, a medida que progresa- ba el experimento en todos los tratamientos, en los cuales, el $\mathrm{CCL}_{4}$ estaba presente.

El estudio histopatológico realizado en la semana seis mostró vacuolización y fibrosis leve alrededor de la vena central del lobulillo hepático en todos los tratamientos, excepto en el grupo control, que mostró normalidad (Figura 1). Posteriormente, en la semana ocho, se presentaron estas lesiones con mayor intensidad, pero con la misma severidad en todos los tratamientos; durante la semana diez, se observaron vacuolización y fibrosis importantes, con severa reducción del tejido hepático funcional.

Los niveles plasmáticos de albumina en los tratamientos con ACR, leucina, valina e isoleucina estuvieron por debajo del nivel fisiológico $35-40 \mathrm{~g} / \mathrm{dl}$ y no se observó diferencia estadís- 


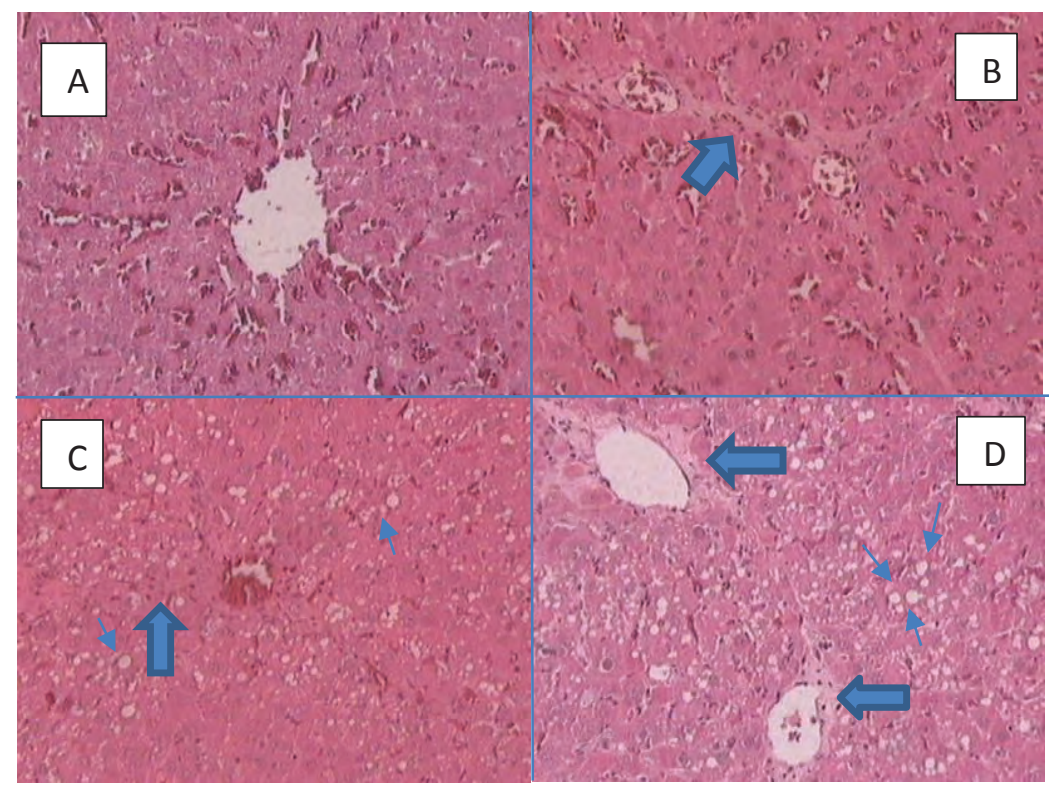

Figura 1: Daño hepático progresivo. A) T5 control en la semana seis, se observa una porción de lobulillo hepático normal con su vena central (400x); B) T0 CCl4 semana seis, fibrosis leve uniendo dos venas centrales (400x), flecha gruesa; C) T0 CCl4 semana ocho, fibrosis (flecha gruesa) y vacuolización (flechas delgadas) moderadas alrededor de central (400x); D) T0 CCl4 semana diez, vacuolización (flechas delgadas) y fibrosis (flechas gruesas) intensas entre dos venas centrales (400x).

tica entre tratamientos. En promedio, el nivel de albúmina estuvo entre $25-30 \mathrm{~g} / \mathrm{dl}$, en todos los tratamientos, excepto para el grupo control, cuyo promedio fue de $35,2 \mathrm{~g} / \mathrm{dl}$.

Se encontraron diferencias significativas $(p<0,05)$, al evaluar las variables consumo de alimentos y peso corporal. Los animales del grupo valina tuvieron un consumo de alimento significativamente mayor, $78 \mathrm{~g} /$ día, en promedio, al compararlos con el T0,65g/día. Además, en cuanto al peso corporal, los tratamientos valina $249 \mathrm{~g}$ y leucina $225 \mathrm{~g}$ presentaron diferencias estadísticamente significativas $(p<0,05)$, al compararlos con el tratamiento T0, 190g.

Excepto la pérdida de peso, no se observaron signos de insuficiencia hepática en las ratas durante el experimento; tampoco, se encontraron lesiones macroscópicas durante las necropsias compatibles con lesión hepática.

Existe un interés especial en establecer si la terapia con aminoácidos de cadena ramificada (ACR) tiene efectos terapéuticos favorables en pacientes que sufren de enfermedad hepática crónica, debido al estado catabólico, que caracteriza esta condición. Los ACR no solo sirven de sustrato en la síntesis proteica, sino que también regulan el catabolismo de estas sustancias. Varios estudios han demostrado el efecto estimulante de los ACR sobre la síntesis de proteínas y la inhibición de la proteólisis (Holecek, 2010; Ishihara et al. 2014; Chen et al. 2015).
En este trabajo, el suplemento de la dieta con valina y leucina mostraron ventajas importantes respecto al equilibrio proteico de las ratas enfermas de hepatitis crónica. El tratamiento con valina reveló un consumo mayor de alimentos, mientras que este mismo tratamiento y el tratamiento leucina presentaron un peso significativamente superior. Y si bien, ambas respuestas son extremadamente importantes para un paciente con daño hepático, no se acompañaron de una mejoría en los parámetros de patología clínica, como el nivel plasmático de albumina y el desarrollo lesiones tisulares. Estos resultados coinciden con lo reportado por Nakaya et al. (2002), Marchesini et al. (2005) y Tajiri \& Shimizu (2013), investigadores quienes observaron que el suministro de ACR en pacientes humanos con cirrosis mejora la condición corporal y el estado nutricional, lo que se reflejó en una calidad de vida más alta y supervivencia mayor.

También, Chen et al. (2015) encontraron que los ACR mejoran la síntesis de proteína hepática, lo que ayuda a corregir el estado catabólico del paciente con daño hepático y disminuye la presentación de complicaciones secundarias, como el sangrado gastrointestinal. Al parecer, todo lo anterior se debe a que los ACR escapan del metabolismo hepático y están disponibles en la circulación general, para la síntesis de proteínas (Marchesini et al. 2005).

Tradicionalmente, los trabajos en los cuales se suplementan ACR en pacientes con daño hepático utilizan estos compues- 
tos, de forma conjunta (Usui et al. 1996, Marchesini et al. 2005); sin embargo, Tomiya et al. (2002) demostraron en un estudio in vitro que la leucina era superior a la valina y la isoleucina, en estimular la secreción del factor de crecimiento hepático, por las células estrellas, factor que estimula la producción de albumina, por los hepatocitos y su proliferación in vitro e in vivo (Tomiya et al. 2002; Tajiri \& Shimizu, 2013).

A luz de los hallazgos de Tomiya et al. (2002), se podría pensar que los pacientes cirróticos se benefician del suplemento de leucina, más que de isoleucina y valina; no obstante, en este trabajo, el tratamiento con valina presentó una diferencia significativa en el consumo de alimentos y una ganancia de peso mayor, que los demás tratamientos.

El nivel plasmático de albumina no rebasó el límite inferior normal en ningún tratamiento, excepto en el control, en contraste con lo reportado por Kajiwara et al. (1998), quienes observaron que el nivel plasmático de albúmina se mantenía dentro de los rangos fisiológicos en ratas, a las cuales, se indujo daño hepático con $\mathrm{CCL}_{4}$, tratadas con ACR. De otra parte, Ishihara et al. (2014) y Chen et al. (2015) reportaron que el suplemento con ACR a personas que padecían cirrosis o carcinoma hepático presentaron incrementos en el nivel de albumina plasmático.

Una concentración muy baja de albúmina produce disminución de la presión oncótica, lo que trae, como consecuencia, el desarrollo de ascitis y edema periférico (Brunton et al. 2009). En este experimento, no se advirtieron síntomas relacionados con hipoalbuminemia.

No se observaron diferencias significativas estadísticas en el grado de fibrosis, infiltración celular y vacuolización tisular, entre tratamientos. Ya se había reportado, que el tratamiento con ACR no alteraba la progresión de las lesiones (Kajiwara et al. 1998) y, a pesar de la gravedad de éstas, no se observaron signos relacionados a fallo hepático. De otra parte, el grupo tratado con valina presentó un consumo mayor de alimento y mayor peso corporal y es posible que estos animales tuvieren una supervivencia mayor, si el experimento se hubiera prolongado.

Se concluye que la suplementación dietética con valina y leucina en ratas con daño hepático crónico podría contrarrestar el desbalance calórico-proteico, al estimular el consumo de alimento y promover un mejor peso corporal. Esta recomendación podría estar indicada en otras especies animales y el hombre, especialmente, porque la suplementación con estos aminoácidos no origina complicaciones.

Conflictos de interés: El manuscrito fue preparado y revisado con la participación de todos los autores, quienes declara- mos que no existe conflicto de intereses que ponga en riesgo la validez de los resultados presentados.

\section{BIBLIOGRAFÍA}

\section{AMERICAN VETERINARY MEDICAL ASSOCIATION.} Guidelines on euthanasia. 2007. Disponible desde Internet en: www.avma.org/resources/euthanasia. pdf (con acceso 10/10/15).

2. BRUNTON, L.; LAZO, J.; PARKER, K. 2009. Las bases farmacológicas de la terapéutica. Goodman \& Gilman. 11ed. México. McGrawHill. p.567-569.

3. CHEN, L.; CHEN, Y.; WNG, X.; LI, H.; ZHANG, H.; GONG, J.; SHEN, S.; YIN, W.; HU, H. 2015. Efficacy and safety of oral branched-chain amino acid supplementation in patients undergoing interventions for hepatocellular carcinoma: a meta-analysis. Nutr. J. USA. 14 (67):1502-1515.

4. CHARTON, M. 2006. Branched-chain amino acid enriched supplements as therapy for liver disease. J. Nutr. USA. 136:295-298.

5. COMMITTE OF AMERICAN INSTITUTE OF NUTRITION -AIN-. 1977. Report of American Institute of Nutrition Ad Hoc Committee on Standards for Nutrition studies. J. Nutr. USA. 107:1340-1348.

6. GARCÍA, M.; GÁLVEZ, J.; ARMENDÁRIZ, J. 2007. Blancos terapéuticos para revertir la cirrosis hepática. Rev. Méd. Chile.135:783-791.

7. HOLECEK, M. 2010. Three targets of branched-chain amino acid supplementation in the treatment of liver disease. Nutr. USA 26:482-490.

8. ISHIHARA, T.; IWASA, M.; TANAKA, H.; KAITO, M.; IKOMA, J.; SHIBATA, T.; TAKEI, Y. 2014. Effect of branched-chain amino acids in patients receiving intervention for hepatocellular carcinoma. China. 20 (10): 2673-2680

9. KAJIWARA, K.; OKUNO, M.; KOBAYASHI, T.; HONMA, N.; MAKI, T.; KATO, M.; OHNISHI, H.; MUTO, Y.; MORIWAKI, H. 1998. Oral supplementation with branched-chain amino acids improves survival rate of rats with carbon tetrachloride-induce liver cirrhosis. Digest. Dis. Sci. 43:1572-1579.

10. KHANNA, S.; GOPALAN, S. 2007. Role of branchedchain amino acids in liver diseases: the evidence for and against. Curr. Opin. Clin. Nutr. 10:297-303. 
11. KONDRUP, J. 2006. Nutrition in end stage liver disease. Best Pract. Res. Cl. Ga. ENGLAND 20:547-560.

12. LARSEN, F. 2008. Branched-chain amino acids antagonism in the patients with cirrhosis and a simulated upper GI bleed. J. Hepatol. ENGLAND 49:686-687.

13. MARCHESINI, G.; MARZOCCHI, R.; NOIA, M.; BIANHI, G. 2005. Branched-chain amino acid supplementation in patients with liver diseases. J. Nutr. USA. 135:1596-1601.

14. MORIWAKI, H.; MIWA, Y.; TAJIKA, M.; KATO, M.; FUKUSHIMA, H.; SHIRAKI, M. 2004. Branchedchain amino acids as a protein and energy source in liver cirrhosis. Biochem. Bioph. Res. Co. USA 313:405-409.

15. MUDDU, J.; GUHA, I.; ELSHARKAWY, M.; MANN, A. 2007. Resolving fibrosis in the diseases liver: Translating the scientific promise to the clinic. Int. J. Biochem. Cell B. USA 39: 695-714.

16. NAKAYA, Y.; HARAD, N.; KAKI, S.; OKADA, K.; TAKAHASHI, A.; INOI, J.; ITO, S. 2002. Severe catabolic state after prolonged fasting in cirrhotic patients: effect of oral branched-chain amino-acid enriched nutrient mixture. J. Gastroenterol. JAPAN 379: 531536.

17. NAKAYA, Y.; OKITA, K.; SUZUKI, K.; MORIWAKI, H.; KATO, A.; KAITO, M.; WATANABE, A.; HABU, D., ITO, S.; ISHIKAWA, T.; KAWAMURA, N.; ARAWAKA, Y. 2007. BCAA-enriched snack improves nutritional State of cirrhosis. Nutri. USA 23:113-120.
18. PROCTOR, E.; CHATAMRA, K. 1984. Standardized micronodular cirrhosis in the rat. Eur. Surg. Res. SWITZERLAND 16:182-186.

19. TAJIRI, K.; SHIMIZU, Y. 2013. Branched-chain amino acids liver diseases. World J. Gastroenterol. China. 19(43):7620-7629.

20. THEISE, N. 2007. Liver biopsy assessment in chronic viral hepatitis. Mod. Pathol. USA. 20:3-14.

21. TOMIYA, T.; INOUE, Y.; YANASE, M.; ARAI, M.; IKEDA, H.; TEJIMA, K.; NAGASHIMA, K.; NISHIKAWA, T.; FIJIWARA, K. 2002. Leucine stimulates the secretion of hepatocyte grow factor by hepatic stellate cells. Biochem. Bioph. Res. Co. USA. 297:1108-1111.

22. USUI, T.; MORIWAKI, H.; HATAKEYAMA, H.; KASAI, T.; KATO, M.; SEISHIMA, M.; OKUNO, M.; YOSHIDA, T.; MUTO, Y. 1996. Oral supplementation with branched-chain amino acids improves tansthyretin turnover in rats with carbon tetrachloride induced liver cirrhosis. J. Nutri. USA. 126:1412-1420.

23. YOSHIDA, T.; MUTO, Y.; MORIWAKI, H.; YAMATO, M. 1989. Effect of long-term oral supplementation with branched-chain amino acid granules on the prognosis of liver cirrhosis. Gastroenterol. Jpn. 24:692-698.

Recibido: Febrero 19 de 2016

Aceptado: Mayo 2 de 2016

Cómo citar:

Cruz Amaya, J.M.; Flórez Cuadros, M.; Narváez Solarte, W. 2016. Suplementación con aminoácidos de cadena ramificada en la progresión de la hepatitis crónica. Rev. U.D.C.A Act. \& Div. Cient. 19(1): 155-160. 